\title{
Kasabach-Merritt syndrome
}

\author{
INSERM
}

\section{Source}

INSERM. (1999). Orphanet: an online rare disease and orphan drug data base. KasabachMerritt syndrome. ORPHA:2330

Kasabach-Merritt syndrome (KMS), also known as hemangioma-thrombocytopenia syndrome, is a rare disorder characterized by profound thrombocytopenia, microangiopathic hemolytic anemia, and subsequent consumptive coagulopathy in association with vascular tumors, particularly kaposiform hemangioendothelioma or tufted angioma. 